\title{
Metallic wear debris collected from patients induces apoptosis in rat primary osteoblasts via reactive oxygen species-mediated mitochondrial dysfunction and endoplasmic reticulum stress
}

\author{
FEI YANG ${ }^{1 *}$, JIAN TANG $^{1}$, KERONG DAI $^{1}$ and YAN HUANG ${ }^{1,2 *}$ \\ ${ }^{1}$ Shanghai Key Laboratory of Orthopedic Implant, Department of Orthopedics, Shanghai Ninth People's Hospital, \\ Shanghai Jiao Tong University School of Medicine, Shanghai 200011; ${ }^{2}$ Shanghai Geriatric Institute of Chinese Medicine, \\ Shanghai University of Traditional Chinese Medicine, Shanghai 200031, P.R. China
}

Received June 22, 2018; Accepted October 31, 2018

DOI: $10.3892 / \mathrm{mmr} .2019 .9825$

\begin{abstract}
Although total hip arthroplasty is considered to be an effective surgical procedure for treating hip joint diseases, it is hindered by implant wear debris, which induces aseptic loosening. Various cell types are involved in this pathogenesis; however, the interactions between wear debris and osteoblasts, which serve a crucial role in bone formation, have not been clearly illustrated. In the present study, minor metallic wear particles were collected from the interfacial membrane around loosened implants of patients, and the biological effects of these particles on rat primary osteoblasts were then explored. The results demonstrated that metallic wear debris was able to induce the apoptosis of treated cells in a concentrationand time-dependent manner. Furthermore, it was identified that reactive oxygen species (ROS) generation increased, the mitochondrial membrane potential collapsed, and the mitochondria-caspase-dependent and endoplasmic reticulum (ER) stress apoptotic pathways were activated following metallic wear debris application. In addition, apoptosis and associated pathways were inhibited by the use of $\mathrm{N}$-acetyl-L-cysteine, an antioxidant that suppresses ROS production, indicating that the ROS generation triggered ER stress, mitochondrial dysfunction and downstream cascades that contributed to cell apoptosis. These findings suggest that metallic wear debris-induced ROS serve an important role in the apoptosis of osteoblasts. This provides a valuable insight, not only into
\end{abstract}

Correspondence to: Dr Fei Yang or Dr Yan Huang, Shanghai Key Laboratory of Orthopedic Implant, Department of Orthopedics, Shanghai Ninth People's Hospital, Shanghai Jiao Tong University School of Medicine, 639 Zhizaoju Road, Shanghai 200011, P.R. China E-mail: feiyangyn@163.com

E-mail: huangyan1113@163.com

${ }^{*}$ Contributed equally

Key words: metallic wear debris, apoptosis, reactive oxygen species, endoplasmic reticulum stress, mitochondrial dysfunction understanding the mechanisms underlying the involvement of osteoblasts in osteolysis, but also into a potential novel therapeutic approach to treat implant aseptic loosening.

\section{Introduction}

Total hip arthroplasty is considered to be one of the most effective therapeutic approaches for hip joint diseases (1). It can effectively treat late/severe arthritis, relieve pain and restore joint function. However, with increasing life expectancy, hip joint replacements must last longer, and the problem of prosthetic loosening is becoming increasingly prominent (2). As a result, revision arthroplasty procedures are increasingly required. Revision surgery is expensive, time-consuming and dangerous for the patient, and the results are not as good as for primary arthroplasties (3). Studies have indicated that 5-20\% of prostheses are affected by aseptic loosening, which is now widely believed to be induced by wear debris generated from prosthetic implant materials (4-6).

Subsequent to implantation of hip prosthesis, wear debris may be produced between prosthetic implants and the bone interface due to micro-movement and friction. The wear debris activates numerous cell types, including fibroblasts, monocytes, macrophages, giant cells, osteocytes, osteoblasts and osteoclasts $(7,8)$, which then induce chronic inflammatory responses and the release of cytokines and chemokines in the periprosthetic soft tissue $(9,10)$. These inflammatory factors and reactions hamper bone formation by decreasing the synthesis of type I collagen, the major component of bone matrix, and elevating the levels of matrix-degrading enzymes $(11,12)$. In addition, bone resorption is enhanced via activation of the adhesion and differentiation of osteoclasts (13). Overall, the phagocytosis of wear particles by various cells disrupts the balance between bone formation and resorption in the bone remodeling process, potentially leading to periprosthetic osteolysis (14).

In addition to the inflammation responsible for wear debris-induced osteolysis, the apoptosis of several associated cell types has recently been demonstrated to be one of the main events involved in wear particle-associated bone resorption $(4,8,15,16)$. For instance, Wang et al (17) reported that direct exposure to commercially pure $\mathrm{Ti}$ and $\mathrm{ZrO}_{2}$ 
particles compromises the viability of human mesenchymal stem cells through the induction of apoptosis in a manner dependent on material composition, particle dosage and time. Landgraeber et al (18) also detected apoptosis of T-lymphocytes in the capsules and interface membranes of patients with aseptic hip implant loosening. Furthermore, Zhang et al (8) demonstrated that the $\beta$-tricalcium phosphate wear particles caused osteocyte dysfunction by mediating apoptotic death and protein kinase B inactivation of osteocytes. In our previous study, the apoptosis of macrophages was identified in the interface membrane of patients undergoing revision surgery for aseptic loosening of hip joint prostheses (4). As the main cells involved in bone formation, osteoblasts have also been reported to be associated with improper bone remodeling around the prosthesis site due to a decrease in their proliferation and an increase in their apoptosis $(13,14)$. However, the specific mechanism underlying the interaction of wear debris and osteoblasts has not been closely investigated (19).

Depending on different stimuli, apoptosis can be executed via extrinsic and intrinsic pathways, which both involve mitochondrial injury and endoplasmic reticulum (ER) stress (20). Certain apoptotic stimuli induce reactive oxygen species (ROS) release, resulting in a drop in MMP, which causes pro-apoptotic factors to move from the intermembrane space into the cytosol and activates apoptosis (21). In a number of recent studies, ROS release has also been demonstrated to induce ER stress, which leads to unfolded protein response (UPR), ER overload response and caspase-12-dependent apoptosis $(22,23)$. Our previous study further demonstrated that the mitochondria-caspase-dependent pathway and ER stress pathway were involved in the process of macrophage apoptosis in the capsules of patients with aseptic loosening (4).

However, the specific mechanisms of ROS generation, mitochondrial injury and ER stress, as well as the roles that they serve in wear debris-induced osteoblast apoptosis and aseptic loosening, are not well understood. Therefore, in the current study, the direct biological effects of metallic wear debris from loosened implants on osteoblasts were investigated, including apoptosis, ROS release, mitochondrial injury and ER stress. The aim of the current study was to expand our understanding of the mechanism underlying metallic wear debris-induced aseptic loosening.

\section{Patients and methods}

Patients. The current study was approved by the Ethics Committee of Shanghai Ninth People's Hospital affiliated to Shanghai Jiao Tong University School of Medicine (Shanghai, China). Written informed consents were obtained from three patients for inclusion and participation prior to commencing the present study. In total, 3 patients with aseptic loosening of hip joint prostheses were included in the study, and none of the included patients had any infections. The patients included 1 male and 2 females, and all exhibited definite aseptic loosening signs under X-ray scanning. The male patient was 63 years old, had an initial diagnosis of traumatic arthritis of the hip joint, and the implantation was conducted 27 years before the revision surgery. One female patient was 60 years old with an initial diagnosis of femoral neck fracture, and underwent implantation 8 years before the revision surgery.
The other female patient was 72 years old with an initial diagnosis of developmental dislocation of the hip, and underwent implantation 15 years before the revision surgery. In each case, the articulations and coupling of the loosened prostheses both comprised a metal femoral head and a polyethylene cup. The tissue around the osteolytic region was obtained from the three patients at the time of revision surgery and used to extract metallic particles.

Particle extraction and characterization. Approximately $5 \mathrm{~g}$ tissue was cut into pieces of $\sim 1 \mathrm{~mm}$ diameter, and repeatedly washed with deionized water to remove blood clots. Next, the tissue was placed into a clean glass container, and $30 \mathrm{ml}$ degreasing mixture (chloroform to methanol, 2:1) was added. Subsequent to shaking at room temperature overnight, $12 \mathrm{ml}$ of $5 \mathrm{M} \mathrm{NaOH}$ solution was added for 48 -h digestion at $65^{\circ} \mathrm{C}(24)$. The digestion solution was subjected to sonication for $10 \mathrm{~min}$ after cooling to room temperature. A 50\% sucrose solution was then added to the digested sample and centrifuged at $3,792 \mathrm{x} \mathrm{g}$ for $1 \mathrm{~h}$ at room temperature. The lower particles were harvested, and deionized water was added for sonication for $5 \mathrm{~min}$ at $80^{\circ} \mathrm{C}$ in order to remove residual sucrose. Subsequently, the metal particles were further separated by density gradient centrifugation at 3,792 $\mathrm{xg}$ for $1 \mathrm{~h}$ at room temperature (25). The pellet suspension was then centrifuged at $474 \mathrm{x} \mathrm{g}$ for $4 \mathrm{~min}$ at room temperature, and the supernatant was collected and resuspended again. This process was repeated three times to collect all supernatants. The collected liquid was centrifuged twice at $948 \mathrm{x} \mathrm{g}$ for $40 \mathrm{~min}$ at room temperature, and the supernatant was discarded. Sonication was used to prevent the aggregation of particles prior to each centrifugation step, and the final collection of particles was stored at $4^{\circ} \mathrm{C}$.

The collected metallic particles were subjected to quantitative analysis of elemental composition using inductively coupled plasma spectroscopy (Agilent 725 ICP-OES; Agilent Technologies, Inc., Santa Clara, CA, USA). The size and distribution of the metallic particles was analyzed using a laser particle size analyzer (Mastersizer 2000; Malvern Panalytical Ltd., Malvern, UK). The final collected metallic particles were sterilized at $180^{\circ} \mathrm{C}$ for $6 \mathrm{~h}$, followed by treatment with $70 \%$ ethanol for $48 \mathrm{~h}$ (26). Subsequently, the metallic wear debris was sonicated for 30 min using a SB3200 Ultrasonic Generator (Shanghai Branson Ultrasonic Co., Ltd., Shanghai, China), prior to incubation with cells. The concentrations of metallic particles used for cell incubation were 0.05 and $0.1 \mathrm{mg} / \mathrm{ml}$.

Cell culture and treatment. The study was approved by the Ethics Committee of Shanghai Ninth People's Hospital affiliated to Shanghai Jiao Tong University School of Medicine. A total of 10 newborn $(<24 \mathrm{~h})$ Sprague-Dawley rats (5 male and 5 female, weighing 5-6 g) were purchased from the Shanghai Model Organisms Center, Inc. (Shanghai, China). Animals were housed under specific pathogen-free conditions at $26^{\circ} \mathrm{C}$, under a 12 -h light/dark cycle, and with free access to sterile food and water. Primary osteoblasts were isolated from the calvarias, as previously described with modifications (27,28). Briefly, neonatal rat calvarias were dissected from adherent soft tissue and washed in a PBS solution. The tissue was cut into pieces using ophthalmic scissors and then 
sequentially digested with $1 \%$ trypsin for $10 \mathrm{~min}$, followed by addition of $0.2 \%$ collagenase type $\approx \mathrm{II}$ for $60 \mathrm{~min}$, in order to release the cells. The cells from the final digestion were resuspended in culture medium and plated in flasks. All the cells were routinely cultured in high-glucose Dulbecco's modified Eagle's medium (DMEM; Hyclone; GE Healthcare Life Sciences, Logan, UT, USA), supplemented with 10\% FBS (Gibco; Thermo Fisher Scientific, Inc., Waltham, MA, USA) and $1 \%$ penicillin-streptomycin in a humidified atmosphere with $5 \% \mathrm{CO}_{2}$ and $95 \%$ humidity at $37^{\circ} \mathrm{C}$ in an incubator. The medium was changed every other day, and cells in the third passage were used for all experiments. In order to evaluate the role of wear debris-induced ROS on apoptosis, cells were treated with $0.1 \mathrm{mg} / \mathrm{ml}$ wear debris for $24 \mathrm{~h}$ in the absence or presence of $50 \mathrm{mM}$ antioxidant $\mathrm{N}$-acetyl-L-cysteine (NAC; Sigma-Aldrich; Merck KGaA, Darmstadt, Germany) dissolved in deionized water. Cells cultured in medium alone were used as the control group.

Analysis of apoptosis by flow cytometry. Annexin V and propidium iodide (PI) double staining was used to determine the rate of apoptosis, using the Annexin V/Dead Cell Apoptosis kit (Invitrogen; Thermo Fisher Scientific, Inc.). Briefly, following treatment with wear debris at 0.05 and $0.1 \mathrm{mg} / \mathrm{ml}$ for 12,24 and $48 \mathrm{~h}$, the primary osteoblasts were harvested and centrifuged at $948 \times \mathrm{g}$ for $5 \mathrm{~min}$ at room temperature. Subsequent to discarding the supernatant, the cell pellets were washed twice with cold PBS and resuspended in $100 \mu$ l Annexin V binding buffer. Next, $5 \mu$ l Alexa Fluor ${ }^{\circledR} 488$ Annexin V and $1 \mu \mathrm{PI}(100 \mu \mathrm{g} / \mathrm{ml})$ staining of the suspension was conducted at room temperature for $15 \mathrm{~min}$, followed by addition and gentle mixing with $400 \mu \mathrm{l}$ Annexin $\mathrm{V}$ binding buffer. Flow cytometry (FACSAria; BD Biosciences, Franklin Lakes, NJ, USA) was finally conducted at a wavelength of $488 \mathrm{~nm}$ to analyze the cell apoptosis.

ROS measurement. 2',7'-dichlorofluorescein diacetate (DCFH-DA; Sigma-Aldrich; Merck KGaA) can be deacetylated by intracellular esterase to form non-fluorescent DCFH, which can be oxidized by ROS, resulting in the formation of the fluorescent compound 2',7'-dichloroflorescein (DCF). The fluorescence intensity of DCF is proportional to the amount of ROS produced by the cells $(29,30)$. In the present study, osteoblasts treated with $0.1 \mathrm{mg} / \mathrm{ml}$ wear debris for 6,12 and $24 \mathrm{~h}$ in the absence or presence of the antioxidant NAC were collected, washed once with ice-cold PBS and incubated with DCFH-DA (50 $\mu \mathrm{M}$ final concentration) at $37^{\circ} \mathrm{C}$ for $30 \mathrm{~min}$ in the dark. Next, the cells were washed twice and maintained in $1 \mathrm{ml}$ of PBS. ROS generation was then assessed using a fluorescence microscope (Axio Imager A2; Zeiss AG, Oberkochen, Germany) or a fluorescence plate reader (Tecan Infinite M200; Tecan Group Ltd., Mannedorf, Switzerland) at excitation and emission wavelengths of 485 and $530 \mathrm{~nm}$, respectively.

Determination of mitochondrial membrane potential (MMP) with JC-1 staining. The primary osteoblasts were treated with wear debris for 12 and $24 \mathrm{~h}$. Next, the medium was removed, and cells were washed with PBS and incubated with JC-1 staining working solution (MitoProbe JC-1 assay kit; Thermo Fisher Scientific, Inc.) at $37^{\circ} \mathrm{C}$ for $20 \mathrm{~min}$. Following washing with staining buffer and PBS, the changes in MMP were visualized using a confocal laser scanning microscope (LSM510; Zeiss AG), and images were captured.

Analysis of extracellular activated caspases. Following incubation with wear debris for 24 and $48 \mathrm{~h}$ in the absence or presence of the antioxidant NAC, the amount of caspase-3/9/12 proteins secreted into the culture medium was determined using the corresponding caspase-3/9/12 fluorometric assay kits (BioVision, Inc., Milpitas, CA, USA), according to the manufacturer's protocol. The absorbance was measured at $400 \mathrm{~nm}$ using a microplate reader (Tecan Infinite M200), and activated caspase-3/9/12 levels were quantified from the relevant standard curve. All samples were measured in triplicate, and the experiment was repeated three times independently.

Western blot analysis. Following treatment under different conditions, the cells were harvested and the medium was removed. A nuclear/cytosol fractionation kit and a mitochondria isolation kit (both purchased from BioVision, Inc.) were used to isolate the nuclei and mitochondria, respectively, according to the manufacturer's protocols. Cells lysis was then performed for $30 \mathrm{~min}$ on ice using radioimmunoprecipitation assay lysis buffer and protease inhibitor cocktail (both Beyotime Institute of Biotechnology, Shanghai, China). Following centrifugation of the lysate at $12,000 \mathrm{x} \mathrm{g}$ for $10 \mathrm{~min}$ at $4^{\circ} \mathrm{C}$, the protein in the supernatant was collected, and a bicinchoninic acid assay was performed to measure protein concentrations. Protein lysate samples $(20 \mathrm{mg})$ were resolved by sodium dodecyl sulfate-polyacrylamide gel electrophoresis using $10 \%$ gels, and proteins were then transferred to polyvinylidene difluoride membranes (EMD Millipore, Billerica, MA, USA). Subsequent to blocking nonspecific interactions by incubation with $5 \%$ skim milk for $1 \mathrm{~h}$ at room temperature, the membranes were probed overnight at $4^{\circ} \mathrm{C}$ with specific primary antibodies against cytochrome c(Cyto-c; 1:1,000; cat. no. 11940), glucose-regulated protein 78 (GRP78; 1:1,000; cat. no. 3177), growth arrest DNA damage 153 (GADD153; 1:1,000; cat. no. 2895) and porin (1:1,000; cat. no. 4866; all purchased from Cell Signaling Technology, Inc., Danvers, MA, USA), as well as with GAPDH antibody (1:5,000; cat. no. KC-5G4; Kangcheng, Shanghai, China). Then, membranes were incubated with goat anti-rabbit or goat anti-mouse horseradish peroxidase-conjugated secondary antibodies (1:5,000; cat. no. A0208 or A0216; Beyotime Institute of Biotechnology) for $1 \mathrm{~h}$ at room temperature. The bound antibodies were then visualized using an enhanced chemiluminescence detection system (EMD Millipore).

Statistical analysis. All data were obtained from three or more experiments, and values are presented as the mean \pm standard deviation. Statistical analysis was conducted using SPSS software version 20 (IBM Corp., Armonk, NY, USA). Statistical comparisons were performed using one-way analysis of variance, followed by the Student-Newman-Keul post hoc test. $\mathrm{P}<0.01$ was considered to indicate a statistically significant difference.

\section{Results}

Patients. The representative case of an aseptic loosening patient (63-year-old male) is depicted in Fig. 1. Pre-operative 

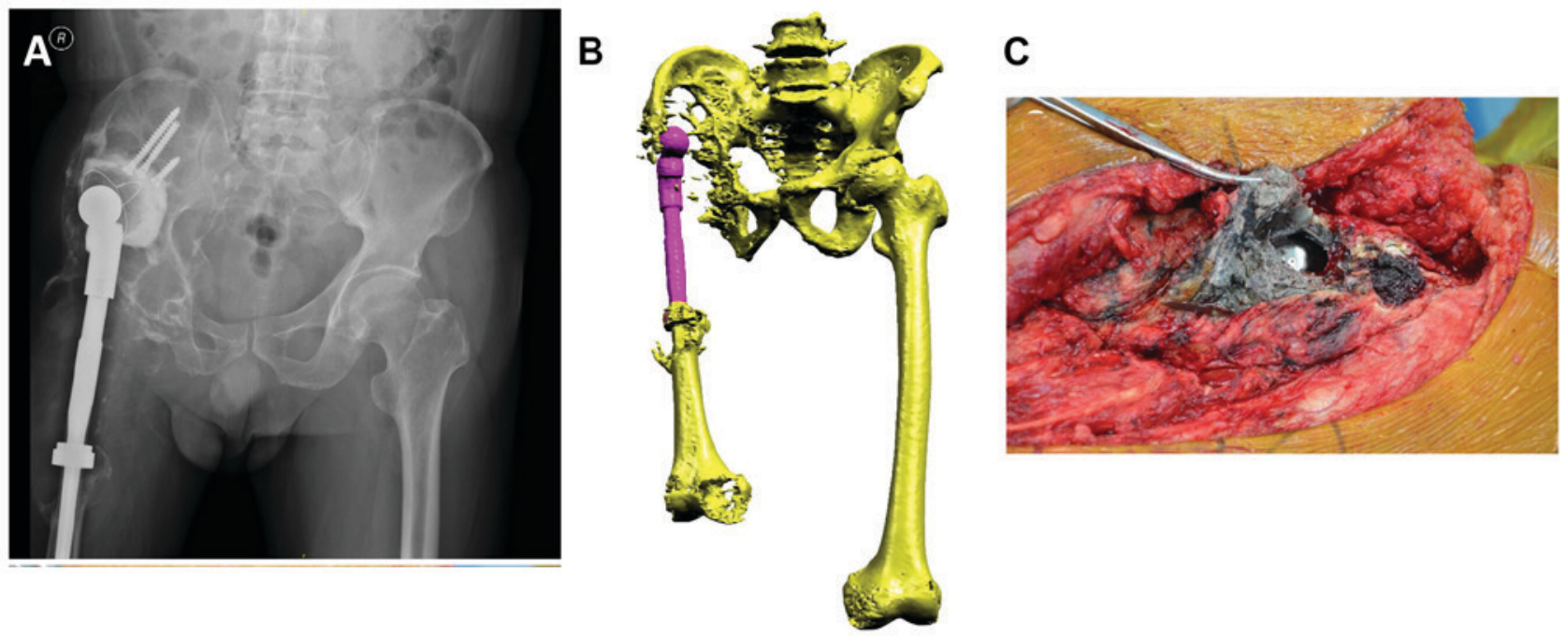

Figure 1. Case of a 63-year-old man with aseptic loosening of hip joint prostheses. (A) Preoperative X-ray and (B) three-dimensional computed tomography scanning indicated migration of the acetabular component and massive periacetabular osteolysis. (C) The tissue around the osteolytic region was black due to severe prosthesis wear and accumulation of metallic wear particles.

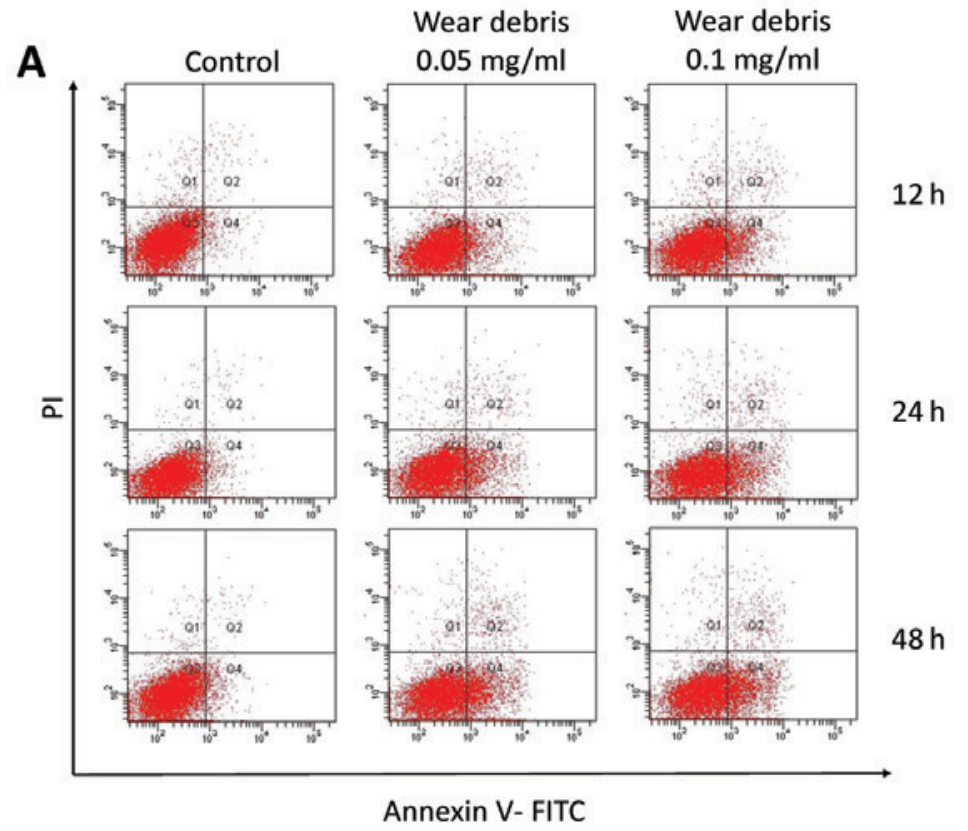

\section{B}

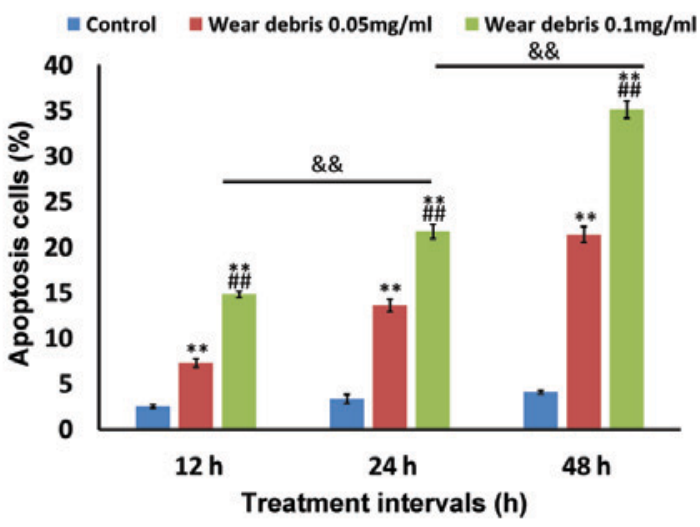

Figure 2. Effect of metallic wear debris on apoptosis induction. Rat primary osteoblasts were treated with wear debris at different concentrations $(0.05$ and $0.1 \mathrm{mg} / \mathrm{ml}$ ) for different time periods (12, 24 and $48 \mathrm{~h}$ ). (A) Flow cytometry was conducted to examine cell apoptosis. Early-apoptotic cells are shown in Q4 (lower right), late-apoptotic cells are shown in Q2 (upper right), and viable cells are shown in Q3 (lower left). (B) Quantitative analysis of the percentage of apoptotic cells. ${ }^{* *} \mathrm{P}<0.01$ vs. control group; ${ }^{\# \#} \mathrm{P}<0.01$ vs. $0.05 \mathrm{mg} / \mathrm{ml}$ group; ${ }^{\&} \mathrm{P}<0.01$. Q, quadrant.

X-ray (Fig. 1A) and three-dimensional computed tomography (Fig. 1B) scans indicated the migration of the acetabular component and massive periacetabular osteolysis. During surgery, the tissue around the osteolytic region appeared black due to the accumulation of a large number of metallic wear particles (Fig. 1C).

Characterization of wear debris. Inductively coupled plasma analysis indicated that the collected metallic wear debris from all three patients were primarily composed of $\mathrm{Ti}, \mathrm{Co}$, $\mathrm{Cr}$, Mo, $\mathrm{Al}$ and $\mathrm{V}$, which was consistent with the prosthesis alloy composition (data not shown). The size of the lower particles following the initial centrifugation ranged between 0.05 and $47.30 \mu \mathrm{m}$, with a mean size of $19.06 \mu \mathrm{m}$. Subsequent to density gradient centrifugation, the final collected metallic particles ranged between 0.45 and $1.67 \mu \mathrm{m}$ in size, with a mean size of $1.22 \mu \mathrm{m}$, and $>90 \%$ of the particles were $0.90-1.50 \mu \mathrm{m}$ in size.

Induction of apoptosis by metallic wear debris at different concentrations in rat primary osteoblasts. The results of flow cytometry analysis indicated that significant apoptosis was induced in rat primary osteoblasts following exposure to metallic wear debris in a concentration- and time-dependent 

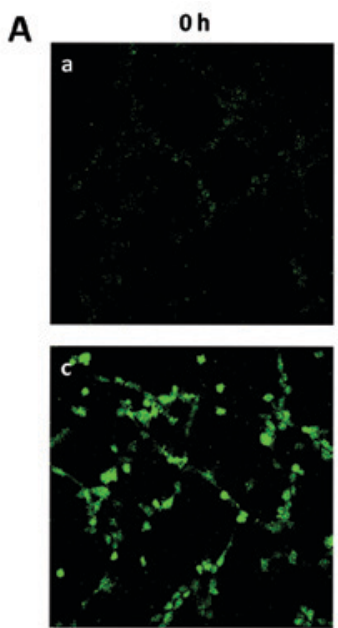

$12 \mathrm{~h}$
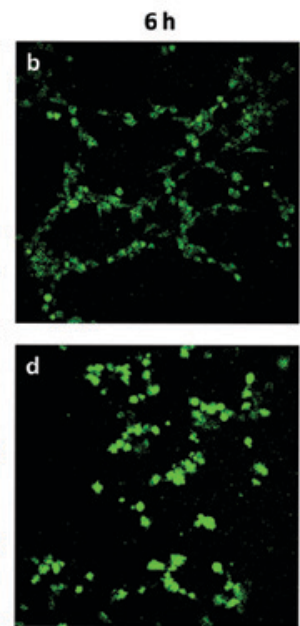

$24 \mathrm{~h}$
B

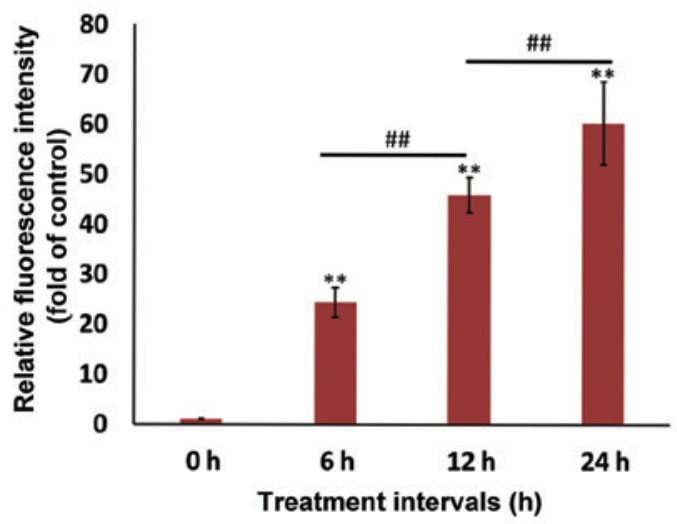

Figure 3. Metallic wear debris-induced apoptosis is associated with ROS generation. (A) Rat primary osteoblasts were treated with metallic wear debris for (a) 0, (b) 6, (c) 12 and (d) $24 \mathrm{~h}$, and images were captured using a fluorescence microscope (magnification, x400). (B) ROS generation was quantified using a fluorescence plate reader. ${ }^{* *} \mathrm{P}<0.01$ vs. $0 \mathrm{~h}$ group; ${ }^{\# \#} \mathrm{P}<0.01$. ROS, reactive oxygen species.
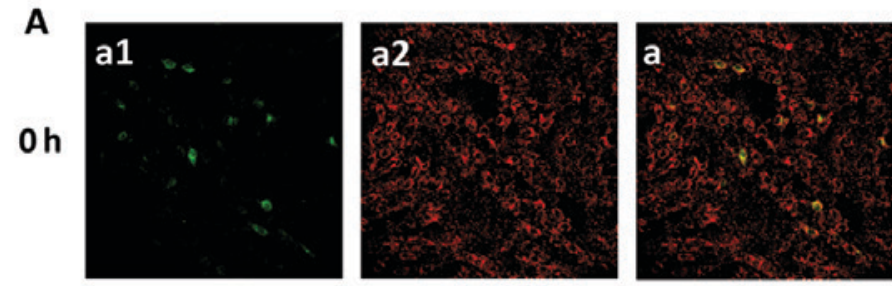

$12 \mathrm{~h}$
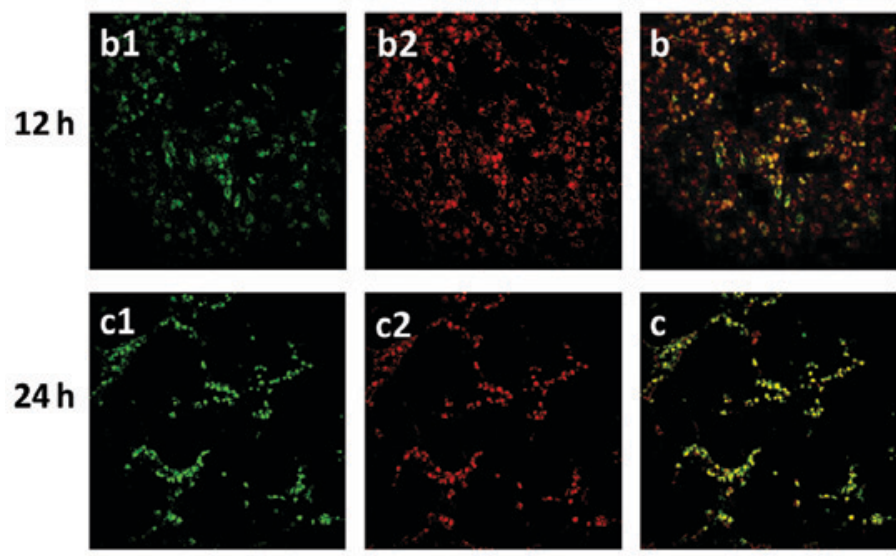

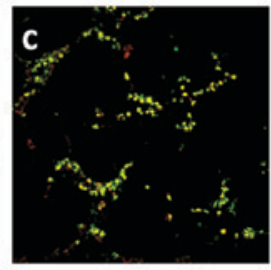

B

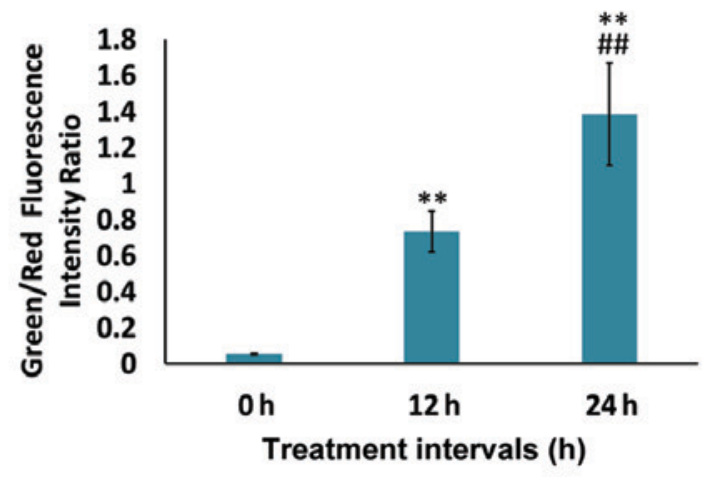

Figure 4. MMP collapses in rat primary osteoblasts following treatment with metallic wear debris. (A) The MMP was determined using a MitoProbe JC-1 assay kit in combination with a laser confocal fluorescence microscope to detect (1) green and (2) red fluorescence (magnification, x200). (B) An increase in the green/red fluorescence intensity ratio indicates mitochondrial depolarization. ${ }^{* *} \mathrm{P}<0.01$ vs. $0 \mathrm{~h}$ group; ${ }^{\# \#} \mathrm{P}<0.01$ vs. $12 \mathrm{~h}$ group. MMP, mitochondrial membrane potential.

manner (Fig. 2). The strongest induction of apoptosis was observed at $0.1 \mathrm{mg} / \mathrm{ml}$ wear debris; therefore, this concentration was selected for use in subsequent experiments.

ROS generation and MMP reduction in metallic wear debris-induced apoptosis. ROS is known to serve an essential role in the regulation of cellular apoptosis (31). As depicted in Fig. 3, ROS release was highly induced by wear debris treatment in a time-dependent manner in rat primary osteoblasts, indicating that the metallic wear debris-induced apoptosis was associated with ROS production. Furthermore, to detect MMP, the JC-1 probe was used, which is a marker of mitochondrial depolarization in the early apoptotic process. During early apoptosis, as a result of the drop in MMP, JC-1 aggregates (red fluorescence) cannot accumulate within the mitochondria and dissipate into JC-1 monomers (green fluorescence), leading to a loss of red fluorescence. Therefore, a decrease in MMP is signified by an increase in the ratio of green to red fluorescence. In the current study, the MMP was reduced in a time-dependent manner following wear debris treatment (Fig. 4), implying that metallic wear debris may mediate the intrinsic apoptotic signaling pathway in osteoblasts.

Metallic wear debris induces apoptosis through the mitochondria-caspase-dependent and ER stress pathways. In addition 


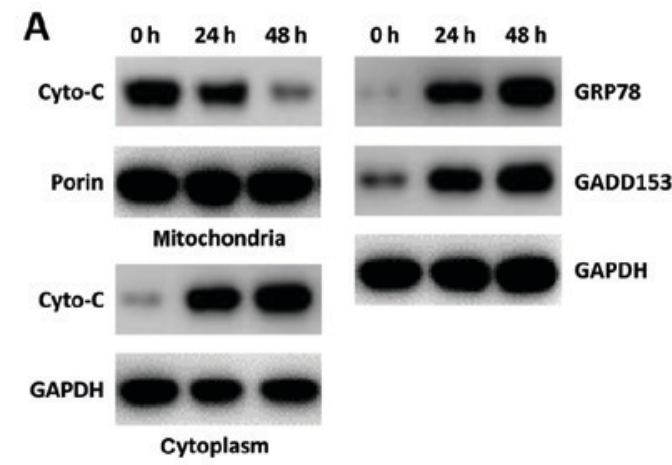

B

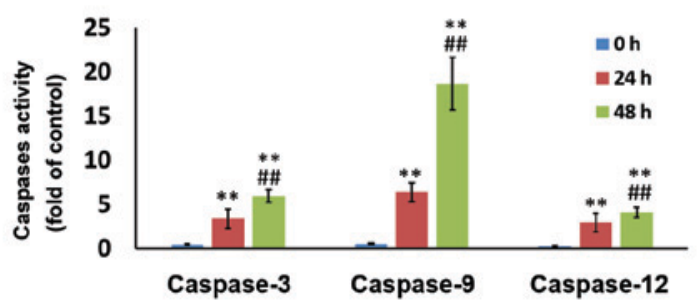

Figure 5. Metallic wear debris results in changes in the expression of proteins and the activation of caspases associated with the mitochondria and ER stress apoptotic pathways. Following wear debris treatment for 24 and $48 \mathrm{~h}$ in rat primary osteoblasts, the expression levels of (A) Cyto-c in the cytoplasm and mitochondria, GRP78 and GADD153, as well as (B) activated caspase-3/9/12 levels, were assessed. ${ }^{* *} \mathrm{P}<0.01$ vs. 0 h group; ${ }^{\# \#} \mathrm{P}<0.01$ vs. $24 \mathrm{~h}$ group. ER, endoplasmic reticulum; Cyto-c, cytochrome c; GRP78, glucose-regulated protein 78; GADD153, growth arrest DNA damage 153.
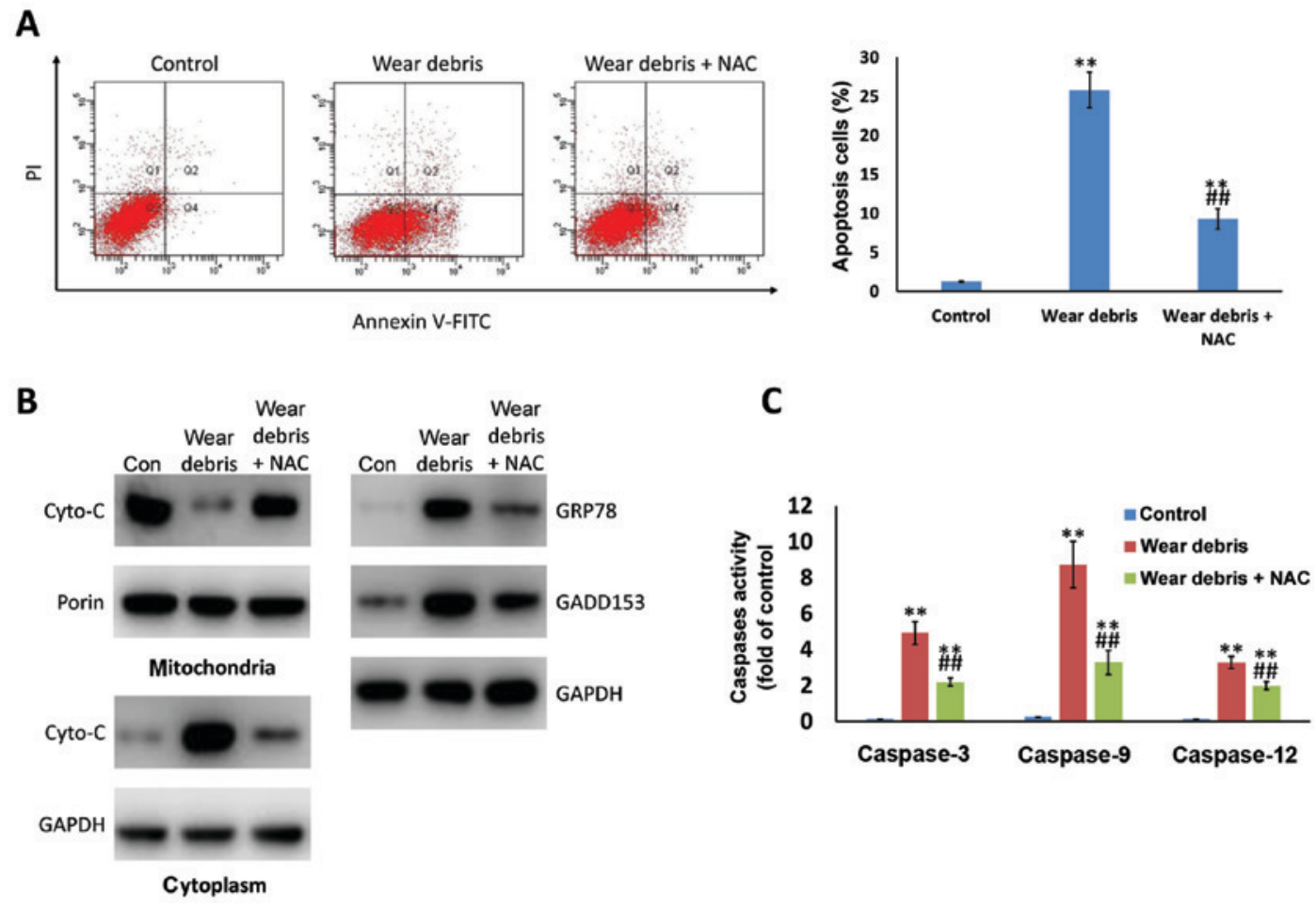

Figure 6. Metallic wear debris induced mitochondria-dependent and ER stress-dependent apoptosis via ROS promotion. Wear debris was used to treat to cells in the presence and absence of NAC for $24 \mathrm{~h}$. (A) Flow cytometry analysis and the quantification of apoptosis rate. (B) Cyto-c levels in the cytoplasm and mitochondria, and expression levels of GRP78 and GADD153. (C) Quantification of activated caspase levels. ${ }^{* *} \mathrm{P}<0.01$ vs. control group; ${ }^{\# \#} \mathrm{P}<0.01$ vs. wear debris group (without NAC). ROS, reactive oxygen species; ER, endoplasmic reticulum; NAC, N-acetyl-L-cysteine; Cyto-c, cytochrome c; GRP78, glucose-regulated protein 78; GADD153, growth arrest DNA damage 153.

to mitochondria-associated apoptosis, ER stress-induced apoptosis also serves a pivotal role in cell death. Western blot analysis indicated that mitochondrial Cyto-c was released into the cytoplasm following wear debris treatment. In addition, the markers of the ER stress apoptotic pathway, namely GRP78 and GADD153, were enhanced by wear debris treatment in a time-dependent manner (Fig. 5A). In addition, activated caspase-3, caspase- 9 and caspase-12 levels were examined, and these were observed to be significantly upregulated following treatment for $48 \mathrm{~h}$ as compared with the value at $24 \mathrm{~h}$ (Fig. 5B). These results suggested that the metallic wear debris induced apoptosis in rat primary osteoblasts by both the mitochondria-caspase-dependent pathway and the ER stress pathway.
Inhibition of ROS attenuates metallic wear debris-induced apoptosis. Antioxidant NAC, as an inhibitor of ROS, was observed to markedly downregulate the metallic wear debris-induced apoptosis (Fig. 6A). In addition, treatment with NAC hindered the increase of Cyto-c in the cytoplasm, and of GRP78 and GADD153 expression levels, as compared with those in cells treated with metallic wear debris alone (Fig. 6B). NAC treatment also significantly suppressed the activation of caspase-3, caspase- 9 and caspase- 12 (Fig. 6C). These findings provided further evidence that the ROS release caused by metallic wear debris resulted in the mitochondria- and ER stress-regulated apoptosis of rat primary osteoblasts. 


\section{Discussion}

As a successful treatment for end-stage arthritis, artificial joint replacement surgery restores mobility, reduces pain and improves the quality of life for millions of patients. However, wear of the implants over time generates debris, which may cause a series of biological responses in the tissues surrounding the joint space, finally leading to aseptic loosening and limiting the longevity of the prosthesis. The wear debris around the prosthesis mainly includes polyethylene (PE), metal and bone cement particles. These particles cause multiple biological reactions, among which metallic particle-induced osteoblast apoptosis has been proposed to be one of the main causes of prosthesis osteolysis in recent years $(14,32,33)$. However, owing to the complexity of the factors involved, the specific regulatory mechanism has not yet been revealed. The current study aimed to investigate the direct biological effects of metallic wear particles on osteoblasts and to elucidate the possible signaling pathways that result in cell dysfunction.

In the majority of studies, the wear debris used for evaluation has been mainly derived from commercial particles $(2,34,35)$. However, due to the various types of wear and the in vivo environment, there are several differences between commercial particles and the wear debris extracted from the interfacial membrane in vivo, including the morphology, size and biological behavior of particles. Therefore, in the present study, metallic wear debris obtained from patients with aseptic joint loosening was used to ensure that the experiments more closely resembled the biological response in vivo and to provide more relevant insights into clinical treatment.

It has been demonstrated that the cellular response to wear debris varies with the size, shape, composition, charge and concentration of particles $(36,37)$. Furthermore, particle phagocytosis represents an important component of the cellular response to implants; thus, the size of wear particles may be a significant factor affecting osteolysis. Studies have estimated that small particles ranging between 0.2 and $10 \mu \mathrm{m}$ in diameter undergo phagocytosis by macrophages (38), and particles $<20 \mu \mathrm{m}$ elicit a significantly greater inflammatory cytokine response (39). In the present study, the wear debris obtained by preliminary extraction included various sizes of particles, which suggested that there may be a variety of wear mechanisms involved. Therefore, density gradient centrifugation was performed to isolate metallic particles of a similar size, which were $<2 \mu \mathrm{m}$ in diameter and within the reported range of phagocytosis.

Wear debris from prostheses has been demonstrated to serve an important role in the initiation of aseptic loosening. Beside the direct effect on bone homeostasis by stimulating osteoclastogenesis, particle exposure also has adverse effects on osteoblastogenesis. Osteoblasts, derived from mesenchymal stem cells, are the main cells in bone tissue and bone formation, and are considered to be critical for prosthesis stability. Continuous exposure to wear debris gives rise to the phagocytosis of mature osteoblasts, and compromises osteoblast function and survival, such as by inducing apoptosis, which finally suppresses bone formation around the prosthesis.

The mitochondrial pathway is considered to be an important apoptotic pathway. Landgraeber et al (40) reported strong expression of Bcl-2-antagonist/killer in macrophages and giant cells in interface tissue, and proposed that the intrinsic apoptotic pathway (mitochondrial pathway) was activated in these cells. As the ER regulates apoptosis, the ER stress pathway has been proposed as another apoptotic pathway that differs from mitochondrial pathways. In our previous study, ER stress pathway-associated markers, including GRP78, GADD153 and caspase-4, were demonstrated to be activated in the aseptic loosening of prostheses using immunohistochemistry (4). It has been demonstrated that ROS are formed as a natural byproduct of the normal metabolism of oxygen, and serve an important role in a series of biological behaviors, such as lipid peroxidation, DNA chain fragmentation and protein modification (41). However, during periods of environmental stress, ROS levels can increase dramatically, resulting in significant damage to cell structures. In the present study, the phagocytosis of metallic wear particles on rat primary osteoblasts directly led to an increase in ROS, which then triggered downstream cellular changes against oxidative stress and finally induced apoptosis. This was also confirmed by the finding that apoptosis was evidently attenuated subsequent to treatment with the antioxidant NAC to inhibit ROS generation. Notably, it has been indicated that ROS can be produced in the ER as a byproduct of oxidative protein folding (42) and in the mitochondria as a byproduct of mitochondrial respiration (43). Furthermore, the ER, mitochondria and oxidative stress interact with each other to interfere with cell function and activate apoptosis signaling in vitro and in vivo $(44,45)$. Hence, in the current study, it was speculated that the stimulation of metallic wear debris induced cellular stress through the activation of ER stress and mitochondrial oxidative stress by ROS generation. In the early stages of ER stress, ROS generation is also associated with the induction of UPR genes, such as GRP78, suggesting the functional propagation of an ER stress response. However, when the UPR process fails to restore adequate ER function, cell death signaling pathways will be activated, as indicated by the activation of GADD153 and caspase-12 (46). By contrast, oxidative stress induces excessive ROS production, activating apoptosis signaling, such as Cyto-c release and caspase cascades, which will contribute to cell apoptosis. In the current study, the aforementioned proteins increased upon metallic wear debris treatment, and their levels were restored by addition of the antioxidant NAC to inhibit ROS production, indicating that wear debris induced-ROS generation was one of the initiating factors of apoptosis.

In conclusion, in the present study, metallic wear debris particles of a similar size were isolated and collected from the tissue around the loosened implants of patients, and these particles were found to directly cause the apoptosis of rat primary osteoblasts. The results indicated that ROS generation induced by metallic wear debris triggered ER stress, mitochondrial dysfunction and downstream caspase cascades, ultimately leading to cell apoptosis. This was further verified by the finding that apoptosis was attenuated following antioxidant treatment. These findings improved our knowledge on the mechanism of metallic wear debris-induced osteoblast apoptosis and may provide a basis for controlling bone destruction during the treatment of implant loosening.

\section{Acknowledgements}

Not applicable. 


\section{Funding}

The present study was supported by grants from the National Nature Science Foundation of China (no. 81301548), the Shanghai Municipal Natural Science Foundation (no. 13ZR1424100), the Natural Science Foundation of Shanghai (no. 16ZR1441700), the Shanghai Jiaotong University 'Cross Research Fund of Medical Engineering' (no. YG2013MS57), and the Fund for Key Disciplines of Shanghai Municipal Education Commission (no. J50206).

\section{Availability of data and materials}

All data generated or analyzed during this study are included in this published article.

\section{Authors' contributions}

FY and YH designed the study and performed the experiments. JT obtained patient tissue for particle extraction, and provided $\mathrm{X}$-ray and three-dimensional CT images. KD contributed to method design and revised the manuscript critically for important intellectual content. All authors read and approved the manuscript.

\section{Ethics approval and consent to participate}

The current study was approved by the Ethics Committee of Shanghai Ninth People's Hospital affiliated to Shanghai Jiao Tong University School of Medicine. Written informed consent was obtained from all patients.

\section{Patient consent for publication}

Not applicable.

\section{Competing interests}

The authors declare that they have no competing interests.

\section{References}

1. Meermans G, Konan S, Das R, Volpin A and Haddad FS: The direct anterior approach in total hip arthroplasty: A systematic review of the literature. Bone Joint J 99-B: 732-740, 2017.

2. Petis S, Howard JL, Lanting BL and Vasarhelyi EM: Surgical approach in primary total hip arthroplasty: Anatomy, technique and clinical outcomes. Can J Surg 58: 128-139, 2015.

3. Sundfeldt M, Carlsson LV, Johansson CB, Thomsen P and Gretzer C: Aseptic loosening, not only a question of wear: A review of different theories. Acta Orthop 77: 177-197, 2006.

4. Yang F, Wu W, Cao L, Huang Y, Zhu Z, Tang T and Dai K: Pathways of macrophage apoptosis within the interface membrane in aseptic loosening of prostheses. Biomaterials 32: 9159-9167, 2011.

5. Wooley PH and Schwarz EM: Aseptic loosening. Gene Ther 11: 402-407, 2004.

6. Cherian JJ, Jauregui JJ, Banerjee S, Pierce T and Mont MA: What host factors affect aseptic loosening after THA and TKA? Clin Orthop Relat Res 473: 2700-2709, 2015.

7. Shimizu S, Okuda N, Kato N, Rittling SR, Okawa A, Shinomiya K, Muneta T, Denhardt DT, Noda M, Tsuji K and Asou Y: Osteopontin deficiency impairs wear debris-induced osteolysis via regulation of cytokine secretion from murine macrophages. Arthritis Rheum 62: 1329-1337, 2010.
8. Zhang Y, Yan M, Yu A, Mao H and Zhang J: Inhibitory effects of $\beta$-tricalciumphosphate wear particles on osteocytes via apoptotic response and Akt inactivation. Toxicology 297: 57-67, 2012.

9. Hallab NJ and Jacobs JJ: Biologic effects of implant debris. Bull NYU Hosp Jt Dis 67: 182-188, 2009.

10. Goodman SB and Ma T: Cellular chemotaxis induced by wear particles from joint replacements. Biomaterials 31: 5045-5050, 2010.

11. Vidovszky TJ, Cabanela ME, Rock MG, Berry DJ, Morrey BF and Bolander ME: Histologic and biochemical differences between osteolytic and nonosteolytic membranes around femoral components of an uncemented total hip arthroplasty. J Arthroplasty 13: 320-330, 1998.

12. Yao J, Cs-Szabó G, Jacobs JJ, Kuettner KE and Glant TT: Suppression of osteoblast function by titanium particles. J Bone Joint Surg Am 79: 107-112, 1997.

13. Vermes C, Chandrasekaran R, Jacobs JJ, Galante JO, Roebuck KA and Glant TT: The effects of particulate wear debris, cytokines, and growth factors on the functions of MG- 63 osteoblasts. J Bone Joint Surg Am 83-A: 201-211, 2001.

14. Lochner K, Fritsche A, Jonitz A, Hansmann D, Mueller P, Mueller-Hilke B and Bader R: The potential role of human osteoblasts for periprosthetic osteolysis following exposure to wear particles. Int J Mol Med 28: 1055-1063, 2011.

15. Posada OM, Gilmour D, Tate RJ and Grant MH: CoCr wear particles generated from $\mathrm{CoCr}$ alloy metal-on-metal hip replacements, and cobalt ions stimulate apoptosis and expression of general toxicology-related genes in monocyte-like U937 cells. Toxicol Appl Pharmacol 281: 125-135, 2014.

16. Thomas V, Halloran BA, Ambalavanan N, Catledge SA and Vohra YK: In vitro studies on the effect of particle size on macrophage responses to nanodiamond wear debris. Acta Biomater 8: 1939-1947, 2012.

17. Wang ML, Tuli R, Manner PA, Sharkey PF, Hall DJ and Tuan RS: Direct and indirect induction of apoptosis in human mesenchymal stem cells in response to titanium particles. J Orthop Res 21: 697-707, 2003.

18. Landgraeber S, von Knoch M, Löer F, Brankamp J, Tsokos M, Grabellus F, Schmid KW and Totsch M: Association between apoptotis and CD4(+)/CD8(+) T-lymphocyte ratio in aseptic loosening after total hip replacement. Int J Biol Sci 5: 182-191, 2009.

19. O'Neill SC, Queally JM, Devitt BM, Doran PP and O'Byrne JM: The role of osteoblasts in peri-prosthetic osteolysis. Bone Joint J 95-B: 1022-1026, 2013.

20. Orrenius S, Gogvadze V and Zhivotovsky B: Mitochondrial oxidative stress: Implications for cell death. Annu Rev Pharmacol Toxicol 47: 143-183, 2007.

21. Kroemer G, Galluzzi L and Brenner C: Mitochondrial membrane permeabilization in cell death. Physiol Rev 87: 99-163, 2007.

22. Sano R and Reed JC: ER stress-induced cell death mechanisms. Biochim Biophys Acta 1833: 3460-3470, 2013.

23. Gorman AM, Healy SJ, Jäger R and Samali A: Stress management at the ER: Regulators of ER stress-induced apoptosis. Pharmacol Ther 134: 306-316, 2012.

24. Minovic A, Milowsev I, Pisot V, Cör A and Antolic V: Isolation of polyacetal wear particles from periprosthetic tissue of isoelastic femoral stems. J Bone Joint Surg Br 83: 1182-1190, 2001.

25. Maloney WJ, Smith RL, Schmalzried TP, Chiba J, Huene D and Rubash H: Isolation and characterization of wear particles generated in patients who have had failure of a hip arthroplasty without cement. J Bone Joint Surg Am 77: 1301-1310, 1995.

26. Liu F, Zhu Z, Mao Y, Liu M, Tang T and Qiu S: Inhibition of titanium particle-induced osteoclastogenesis through inactivation of NFATc1 by VIVIT peptide. Biomaterials 30: 1756-1762, 2009.

27. Wornham DP, Hajjawi MO, Orriss IR and Arnett TR: Strontium potently inhibits mineralisation in bone-forming primary rat osteoblast cultures and reduces numbers of osteoclasts in mouse marrow cultures. Osteoporos Int 25: 2477-2484, 2014.

28. Hou JM, Chen EY, Wei SC, Lin F, Lin QM, Lan XH, Xue Y and Wu M: Lactoferrin inhibits apoptosis through insulin-like growth factor I in primary rat osteoblasts. Acta Pharmacol Sin 35: 523-530, 2014.

29. Zhu WB, Tian FJ and Liu LQ: Chikusetsu (CHI) triggers mitochondria-regulated apoptosis in human prostate cancer via reactive oxygen species (ROS) production. Biomed Pharmacother 90: 446-454, 2017. 
30. Erl W, Weber C and Hansson GK: Pyrrolidine dithiocarbamate-induced apoptosis depends on cell type, density, and the presence of $\mathrm{Cu}(2+)$ and $\mathrm{Zn}(2+)$. Am J Physiol Cell Physiol 278: C1116-C1125, 2000.

31. Sinha K, Das J, Pal PB and Sil PC: Oxidative stress: The mitochondria-dependent and mitochondria-independent pathways of apoptosis. Arch Toxicol 87: 1157-1180, 2013.

32. Haleem-Smith H, Argintar E, Bush C, Hampton D, Postma WF Chen FH, Rimington T, Lamb J and Tuan RS: Biological responses of human mesenchymal stem cells to titanium wear debris particles. J Orthop Res 30: 853-863, 2012.

33. Pioletti DP, Takei H, Kwon SY, Wood D and Sung KL: The cytotoxic effect of titanium particles phagocytosed by osteoblasts. J Biomed Mater Res 46: 399-407, 1999.

34. Yang $X$ and Hutchinson CR: Corrosion-wear of $\beta$-Ti alloy TMZF (Ti-12Mo-6Zr-2Fe) in simulated body fluid. Acta Biomater 42: 429-439, 2016.

35. Cheng T, Zhao Y, Li B, Cheng M, Wang J and Zhang X: Curcumin attenuation of wear particle-induced osteolysis via RANKL signaling pathway suppression in mouse calvarial model. Mediators Inflamm 2017: 5784374, 2017.

36. González O, Smith RL and Goodman SB: Effect of size, concentration, surface area, and volume of polymethylmethacrylate particles on human macrophages in vitro. J Biomed Mater Res 30: 463-473, 1996.

37. Sabokbar A, Pandey R and Athanasou NA: The effect of particle size and electrical charge on macrophage-osteoclast differentiation and bone resorption. J Mater Sci Mater Med 14: 731-738, 2003.

38. Gelb H, Schumacher HR, Cuckler J, Ducheyne P and Baker DG: In vivo inflammatory response to polymethylmethacrylate particulate debris: Effect of size, morphology, and surface area. J Orthop Res 12: 83-92, 1994.
39. Abu-Amer Y, Darwech I and Clohisy JC: Aseptic loosening of total joint replacements: Mechanisms underlying osteolysis and potential therapies. Arthritis Res Ther 9 (Suppl 1): S6, 2007.

40. Landgraeber S, von Knoch M, Löer F, Wegner A, Tsokos M, Hussmann B and Totsch M: Extrinsic and intrinsic pathways of apoptosis in aseptic loosening after total hip replacement. Biomaterials 29: 3444-3450, 2008.

41. Devasagayam TP, Tilak JC, Boloor KK, Sane KS, Ghaskadbi SS and Lele RD: Free radicals and antioxidants in human health: Current status and future prospects. J Assoc Physicians India 52: 794-804, 2004.

42. Santos CX, Tanaka LY, Wosniak J and Laurindo FR: Mechanisms and implications of reactive oxygen species generation during the unfolded protein response: Roles of endoplasmic reticulum oxidoreductases, mitochondrial electron transport, and NADPH oxidase. Antioxid Redox Signal 11: 2409-2427, 2009.

43. Brand MD: The sites and topology of mitochondrial superoxide production. Exp Gerontol 45: 466-472, 2010.

44. Malhotra JD and Kaufman RJ: Endoplasmic reticulum stress and oxidative stress: A vicious cycle or a double-edged sword? Antioxid Redox Signal 9: 2277-2293, 2007.

45. Wang J, Yang X and Zhang J: Bridges between mitochondrial oxidative stress, ER stress and mTOR signaling in pancreatic $\beta$ cells. Cell Signal 28: 1099-1104, 2016.

46. Buytaert E, Matroule JY, Durinck S, Close P, Kocanova S, Vandenheede JR, de Witte PA, Piette J and Agostinis P: Molecular effectors and modulators of hypericin-mediated cell death in bladder cancer cells. Oncogene 27: 1916-1929, 2008.

This work is licensed under a Creative Commons Attribution-NonCommercial-NoDerivatives 4.0 International (CC BY-NC-ND 4.0) License. 